\title{
Effects of the Ar ions pre-amorphization of Si substrate on interface mixing of Fe/Si bilayers
}

\author{
N. BIBIĆ ${ }^{*} \dagger$, V. MILINOVIĆ $*$ M. MILOSAVLJEVIĆ $*$, \\ F. SCHREMPEL $\ddagger$, M. ŠILJEGOVIĆ $† \&$ K.P. LIEB $\dagger$ \\ *VINČA Institute of Nuclear Sciences, Belgrade, Serbia \\ $\dagger$ †I. Physikalisches Institut, Universität Göttingen, Friedrich-Hund-Platz 1, \\ D-37077 Göttingen, Germany \\ $\ddagger$ Fakultät für Physik, Friedrich-Schiller-Universität Jena, D-07743 Jena, Germany
}

Key words. Amorphous semi-conductors, ion beam mixing, RBS, SEM, TEM.

\begin{abstract}
Summary
Ion beam mixing of $\mathrm{Fe} / \mathrm{Si}$ bilayers, induced by $100 \mathrm{keV}$ ${ }^{40} \mathrm{Ar}$ ions at room temperature was investigated. Rutherford backscattering spectroscopy (RBS), atomic force microscopy (AFM), scanning electron microscopy (SEM) and transmission electron microscopy (TEM) were applied for structural characterization. The main focus of this study was on the influence of the substrate structure on interface mixing. The influence of the substrate structure is due to the two classes of irradiated bilayers, Fe thin films deposited on crystalline or preamorphized Si substrates. An about 76\% higher efficiency of atomic transport across the pre-amorphized Fe/a-Si interface as compared to that of $\mathrm{Fe} / \mathrm{c}$-Si bilayers was observed.
\end{abstract}

\section{Introduction}

Tailoring thin films of new materials by ion bombardment of layered structures, as an interesting alternative to physical or chemical vapor co-deposition, pulsed-laser deposition, and other thin film deposition methods, has been the subject of numerous studies. Moreover, the precise control of irradiation parameters along with the selection of suitable materials offers advantages in structuring materials on a nanometre scale, nowadays one of the most relevant technological challenges. In the past, silicide formation via ion beam mixing of metal/Si bilayers was studied in great detail (Reader et al., 1993; Dhar et al., 1996).

The present work outlines the influence of the structural properties of Si substrate on the intermixing processes induced by noble gas ion irradiation. The process of amorphization is important in semi-conductor processing, since Si preamorphization allows the formation of ultra shallow junctions.

Correspondence to: N. Bibić. Tel/fax: +381-11-8066-425; e-mail: natasabi@vin. bg.ac.yu
By contrast, fundamental understanding of the ion beaminduced amorphization process in silicon is of continued interest. In this context the effect of $1.0-\mathrm{keV} \mathrm{Ar}^{+}$-ion preimplantation of the Si substrates was studied. Such treatments may induce the amorphization of the region in proximity of the Si wafer surface, changes of the surface topography and roughness, and lowering the concentration of the contaminants, such as hydrocarbon and oxide layers at the $\mathrm{Fe} / \mathrm{Si}$ interface. The results of these investigations, which were obtained with $100-\mathrm{keV}$ Ar ions, revealed a pronounced enhancement of the mixing rate in the pre-amorphized samples.

\section{Materials and methods}

The $\mathrm{Fe} / \mathrm{Si}$ bilayers were prepared by electron-beam evaporation using the thin film deposition facility at VINCA. The substrates used in this experiment were crystalline and pre-amorphized Si (100) wafers. For one set of the samples labelled $\mathrm{Fe} / \mathrm{a}-\mathrm{Si}$, the Si substrates were bombarded with 1.0$\mathrm{keV} \mathrm{Ar}$ ions. The pre-amorphization process was immediately followed by deposition of Fe thin films inside of the deposition chamber. Another set of bilayers labelled Fe/c-Si was prepared by deposition of Fe thin films on a crystalline Si substrate. The Fe films were deposited on room temperature to the thicknesses of 30-32 nm, at an average deposition rate of $0.1 \mathrm{~nm} / \mathrm{s}$ and a pressure of $1 \times 10^{-4} \mathrm{~Pa}$. The ion irradiations were performed with $100-\mathrm{keV} \mathrm{Ar}^{+}$ions provided by the 500-keV IONAS accelerator facility in Göttingen. All irradiations were performed at room temperature (RT). The ion fluences were in the range from $1 \times 10^{15}$ to $2 \times$ $10^{16}$ ionscm $^{-2}$ and the implanted area was $1 \times 1 \mathrm{~cm}^{-2}$ homogeneously covered by means of an $x-y$ sweeping system. The RBS (Rutherford Backscattering Spectrometry) analyses were performed with a $900-\mathrm{keV} \mathrm{He}^{2+}$ ion beam provided by the 


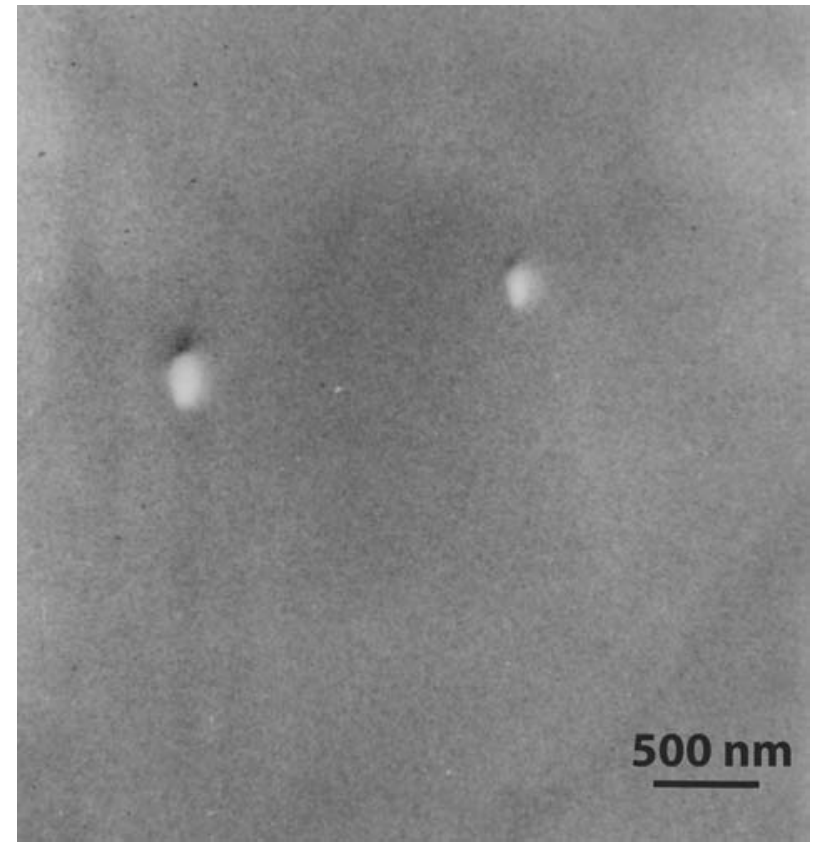

Fig. 1. SEM micrograph taken from the Si substrate bombarded with 1$\mathrm{keV}$ Ar ions.

IONAS facility in Göttingen. Changes of the surface roughness of the samples before and after irradiation were analyzed by atomic force microscopy (AFM). Data were taken in ambient air and in tapping mode. Scans were $1.5 \mu \mathrm{m} \times 1.5 \mu \mathrm{m}, 3 \mu \mathrm{m} \times$ $3 \mu \mathrm{m}$ and $5 \mu \mathrm{m} \times 5 \mu \mathrm{m}$. The surface roughness is represented by the root-mean-square (rms) value. The analysis of surface topography of Si substrate after the pre-amorphization process was performed by scanning electron microscopy (SEM), using a JSM-35 (JEOL) instrument (operated at $25 \mathrm{kV}$ ) (JEOL, Tokyo, Japan). A microstructural characterization was preformed by means of high-resolution cross-sectional transmission electron microscopy (TEM), using Philips EM $400 \mathrm{~T}$ and CM 200 electron microscopes (Philips, Eindhoven, the Netherlands).

\section{Results and discussion}

The surface roughness (rms value) of Fe/a-Si bilayers was deduced from the AFM measurements. With $3 \mu \mathrm{m} \times 3 \mu \mathrm{m}$ scan area we obtained $1.5 \mathrm{~nm}$ rms roughnesses. This implies that the contribution of the surface roughness to the intermixing of the components in subsequent ion beam mixing processes is negligible.

The SEM micrograph in Fig. 1 shows the surface topography of silicon substrate after bombardment with 1.0-keV Ar ions. The Si substrate is flat and uniform, whereas the hillocks in the center correspond to the adsorbed impurities. No changes of the surface topography after pre-amorphization as compared to c-(100) Si substrate were observed.

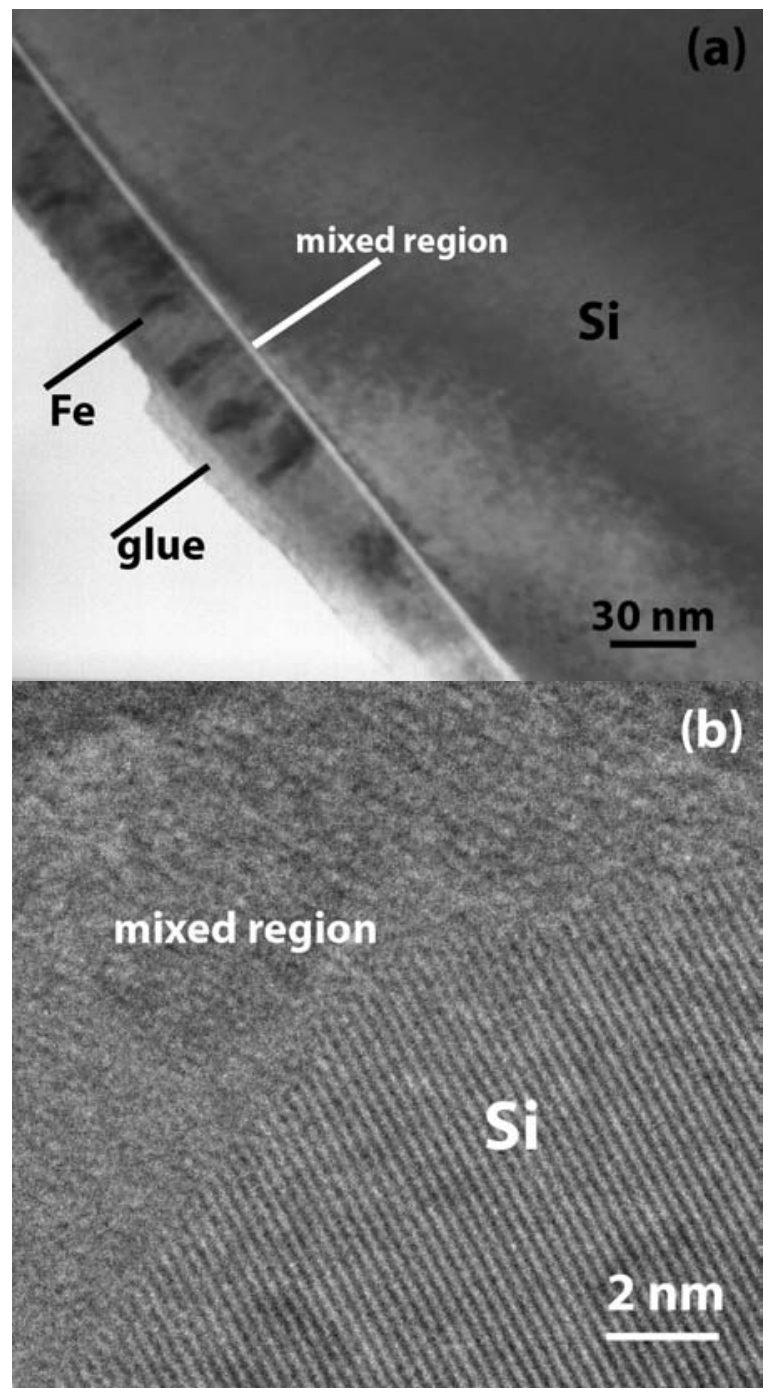

Fig. 2. TEM analysis of a Fe/a-Si sample irradiated with $1 \times 10^{16} \mathrm{Ar}^{+}$ ionscm ${ }^{-2}$ : bright-field image taken from the sample aligned along [110] $\mathrm{Si}$ (a) and high-resolution image taken along [110] Si (b).

Figures 2(a) and (b) show cross-sectional transmission electron microscopy (x-TEM) analysis of a Fe/a-Si sample irradiated with $100-\mathrm{keV} \mathrm{Ar}^{+}$ions to $1 \times 10^{16} \mathrm{~cm}^{-2}$. In (a), the sample was aligned along the [110] Si zone axis. Note the high contrast between the Si substrate and the mixed region, as well as the polycrystalline structure of the top Fe layer. Here we also see a bit of glue on the top of $\mathrm{Fe}$, remaining after sample preparation. The contrast arising from the substrate and the mixed region is nearly equalized, showing that the top $\mathrm{Fe}$ layer and the mixed region are closely bound to the Si substrate. A detailed micro diffraction (MD) analysis for various sample tilts, covering an area of approximately $35 \mathrm{~nm}$ in diameter centred on the intermixed region indicated that the bright-contrast region is amorphous. This leads to the conclusion that the intermixed region has an amorphous 


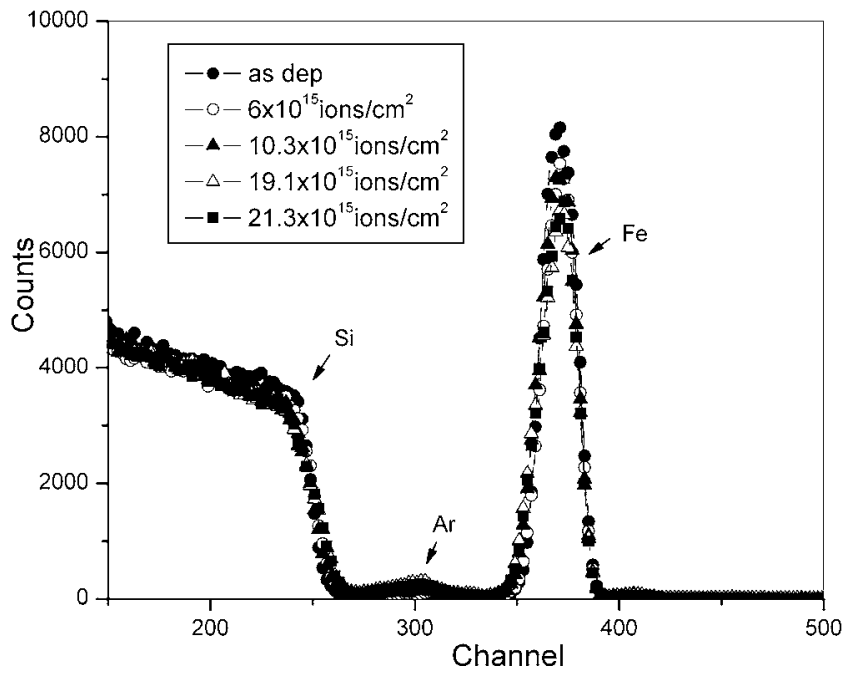

Fig. 3. RBS spectra taken before and after $100-\mathrm{keV} \mathrm{Ar}^{+}$ion irradiations of Fe/a-Si samples.

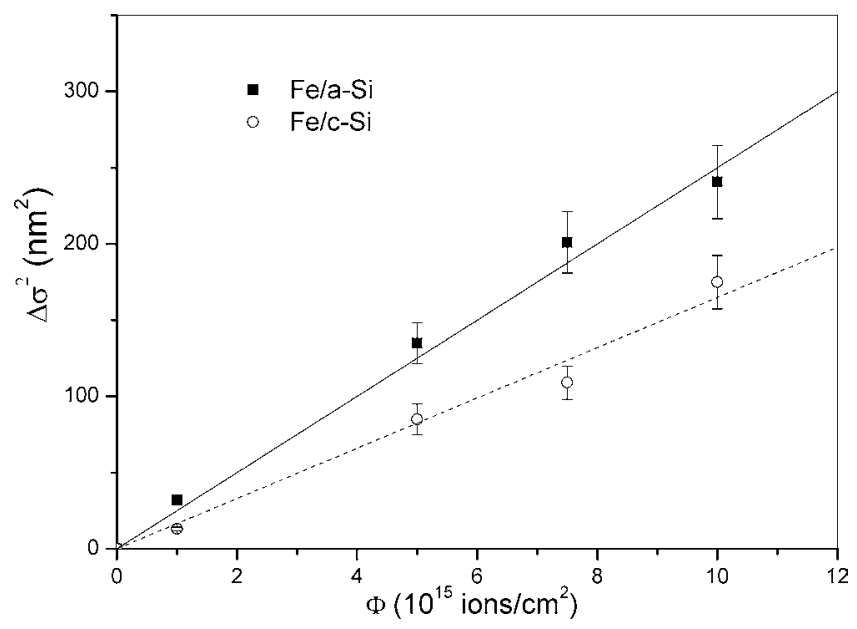

Fig. 4. Interface broadening variance $\Delta \sigma^{2}(\Phi)$ versus ion fluence $\Phi$ for $\mathrm{Fe} / \mathrm{c}-\mathrm{Si}$ and $\mathrm{Fe} / \mathrm{a}-\mathrm{Si}$ bilayers irradiated with $100-\mathrm{keV} \mathrm{Ar}^{+}$ions.

structure. Furthermore, we did TEM analyses using higher magnifications and different sample tilts, which showed no diffraction contrasts within the mixed layer, indicating that this region is fully amorphous. An HRTEM image, taken along [110] Si and shown in Fig. 2(b), exhibits lattice $\{111\} \mathrm{Si}$ fringes from the substrate $(0.313 \mathrm{~nm}$ spacing $)$, and again a different crystalline structure of the mixed region. The analysis of different areas of the sample showed a similar uniform contrast of the intermixed region.

The RBS spectra presented in Fig. 3 were taken from an asdeposited 30-nm Fe/a-Si sample and from samples irradiated with 100-keV $\mathrm{Ar}^{+}$ions at RT. Irradiation fluences were in the range of $0.5-2 \times 10^{16}$ ions $\mathrm{cm}^{-2}$. The spectra clearly show that ion irradiation induces intermixing of the bilayer components at the Fe/a-Si interface. The Ar yield is well separated on the RBS spectra and is located at the metal/silicon interface. On the basis of the Si and Fe depth profiles obtained from RBS, the changes of the variance $\sigma^{2}$ of the Fe and Si concentration profiles were deduced as a function of the ion fluence $\Phi$ providing the mixing rates $k \equiv \Delta \sigma^{2} / \Phi$. Figure 4 illustrates the $\Delta \sigma^{2}$-versus- $\Phi$ plot corresponding to the $\mathrm{Fe} / \mathrm{c}$ $\mathrm{Si}$ and $\mathrm{Fe} / \mathrm{a}-\mathrm{Si}$ bilayers. In both cases, the interface variance $\Delta \sigma^{2}$ varies linearly with the ion fluence $\Phi$. It can be clearly seen that the mixing rate $\left(k \equiv \Delta \sigma^{2} / \Phi\right)$ is higher for the $\mathrm{Fe} / \mathrm{a}-\mathrm{Si}$ bilayers. Since all the other experimental parameters were kept identical for both the Fe/a-Si and Fe/c-Si bialyers, we argue that amorphization of the $\mathrm{Si}$ wafer is the major reason for intermixing enhancement. This can be related to the smaller cooling rate in a-Si $\left(0.3 \times 10^{9} \mathrm{~K} \mathrm{~s}^{-1}\right)$ than that in c-Si $(2.5 \times$ $10^{9} \mathrm{~K} \mathrm{~s}^{-1}$ ) (Schwickert et al., 2003). In the case of interface mixing this may lead to a longer diffusion time and to a higher mixing rate. Consequently, we introduced the ratio of mixing rates for amorphized and crystalline $\mathrm{Si}, R_{\mathrm{a} / \mathrm{c}} \equiv$ $\left[\Delta \sigma^{2} / \Phi\right]_{\mathrm{a}} /\left[\Delta \sigma^{2} / \Phi\right]_{\mathrm{c}}$. For the measured values of $R_{\mathrm{a} / \mathrm{c}}$, we arrived at the weighted mean value of $R_{\mathrm{a} / \mathrm{c}}=1.76(15)$ (Bibić et al., 2007).

\section{Conclusions}

The present careful mixing experiments on $\mathrm{Fe} / \mathrm{Si}$ bilayers with 100-keV Ar ions have given clear evidence of enhanced mixing rates, whenever the Si wafers were pre-amorphized with 1.0$\mathrm{keV}$ Ar ions before deposition of the 30-nm Fe top layer. This enhancement by $76 \%$ relative to crystalline Si wafers may be related to a longer diffusion time in amorphous silicon.

\section{Acknowledgements}

Deutsche Forschungsgemeinschaft has founded this project. Financial support for this work and for project no. 141013 from the Ministry of Science of the Republic of Serbia is gratefully acknowledged.

\section{References}

Reader, A.H., et al. (1993) Transition metal silicides in silicon technology. Rep. Prog. Phys. 56, 1397-1467.

Dhar, S., et al. (1996) Compound formation under local thermal spikes during ion-beam mixing: model and its experimental verification. Phys. Rev. B 54, 5769-5776.

Schwickert, M., et al. (2003) Laser hydriding of crystalline and amorphous silicon. Appl. Phys. A 77, 793-797.

Bibić, N., et al. (2007) Enhanced interface mixing of Fe/Si bilayers on preamorphized silicon substrates. Appl. Phys. Lett. 90, 051901. 